\title{
ACCRETION DISK DYNAMICS OF HER X-1
}

\author{
S. D. VRTILEK ${ }^{1,2}$, F. H. CHENG ${ }^{1}$ \\ 1. University of Maryland, College Park, MD 20742, USA \\ 2. CfA, 60 Garden St., Cambridge, MA 02138, USA
}

\section{Introduction}

The Her $\mathrm{X}-1 / \mathrm{HZ}$ Her system consists of a neutron star with a rotation period of $1.24 \mathrm{~s}$ orbiting a 'normal' stellar companion every $1.7 \mathrm{~d}$. Variation in optical and ultraviolet magnitude is attributed to X-ray heating on the side facing the active neutron star with some contribution from X-ray heating of the disk. The system also has a 35-day period in which the X-ray emission displays so-called main and short 'on' states separated by relative $\mathrm{X}$-ray quiescence. The 1.7-day optical flux variations continue throughout the 35-day period. No clear consensus exists as to the origin of the 35-day period; a popular interpretation is that of occultation of the compact X-ray source by a tilted, precessing, accretion disk.

Each temporal state has its own distinctive spectral behavior. During the main on state the pulse-averaged spectrum is a flat power law with an excess strong thermal component at low energies (a characteristic of many binary X-ray pulsars). Spectral features are detected at $6.4 \mathrm{keV}$ attributed to $\mathrm{Fe} K$-shell, and between $35 \ldots 58 \mathrm{keV}$ attributed to electron-cyclotron resonance implying a surface magnetic field of $(2 \ldots 5) 10^{12} \mathrm{G}$. The pulse profile changes as a function of energy: the pulse peak shifts in phase by $180^{\circ}$ between low and high energies and shows less structure in the low energy band. The spectrum also changes as a function of pulse phase: the soft thermal component and the Fe line flux are strongest during the off-peak of the pulse. This implies that the soft X-rays are likely to be reprocessed hard X-rays and the Fe line emission originates at the reprocessing site.

\section{The 1993 multiwavelength campaign}

The launch of EUVE opened up a new region of the spectrum that is particularly useful for study of the strong soft component. The unusu- 
ally high galactic latitude and correspondingly low interstellar absorption $\left(\mathrm{N}_{\mathrm{H}} \sim 10^{20} \mathrm{~cm}^{-2}\right)$, towards Her $\mathrm{X}-1 / \mathrm{HZ}$ Her make it the only $\mathrm{X}$-ray binary system EUVE is able to study. Observations at other wavelengths are also important since the soft component is likely to be reprocessed radiation; our campaign employed IUE, ROSAT, ASCA, GRO/BATSE, and several ground-based observatories in addition to EUVE.

The campaign was scheduled during the high on state of the 35-day cycle but the X-ray flux observed was a factor of 50 below the expected level (Vrtilek et al. 1994). For the second time in Her X-1's recorded history the system had been caught in a so-called 'anomalous low' state. A substantial, unexpected drop in flux at X-ray energies, with no substantial change in absorbing column density; little or no change in UV and optical fluxes; an increase in pulse period; and no pulsed emission above $1.0 \mathrm{keV}$ are some characteristics of the anomalous low state. With the exception of the increase in pulse period and the degree of X-ray flux reduction these characteristics are similar to those observed during 'normal' 35-day low states. Our observations are particularly exciting because the simultaneous multiwavelength coverage enables us to understand the 'lost' X-ray flux.

The ROSAT pulse profile and energy dependence show the normal main on state behavior during the highest X-ray flux observed (a factor of 2 below the expected): a shift of pulse phase by about $180^{\circ}$ at $0.9 \mathrm{keV}$ is clearly detected. The pulse period measured by ROSAT, $1.237749(1) \mathrm{s}$, shows a sharp increase $(7 \mu \mathrm{s})$ from the previous measurement contrary to the usual spin-up. During the lowest X-ray flux observed neither ROSAT nor ASCA detected pulsed emission above $0.9 \mathrm{keV}$.

Long term observations of Her X-1 by BATSE suggest that the hard $\mathrm{X}$-ray flux of Her X-1 undergoes episodes of reduction that are correlated with spin-down. The correlation between times of low hard X-ray luminosity and episodes of spin-down are predicted by models such as those of Ghosh \& Lamb (1979); an increase in mass accretion rate $(\dot{M})$ causes the Alfven radius at which material joins the magnetosphere to move inward, thereby reducing the drag-producing torques of the field lines threading the disk outside the corotation radius, leading to spin-up.

\section{Modelling of the UV and optical continuum}

The model used to fit the IUE data is described in detail by Cheng, Vrtilek \& Raymond $(1995 ; 1996)$. We adopt the system geometry of Howarth \& Wilson (1983) where changes in X-ray, UV and optical flux during the 35 -day cycle are due to a tilted, precessing, accretion disk. The X-ray heating code is adapted from one used to model the UV flux of low-mass X-ray binaries (LMXBs; Vrtilek et al. 1990). A major difference is that, while the 


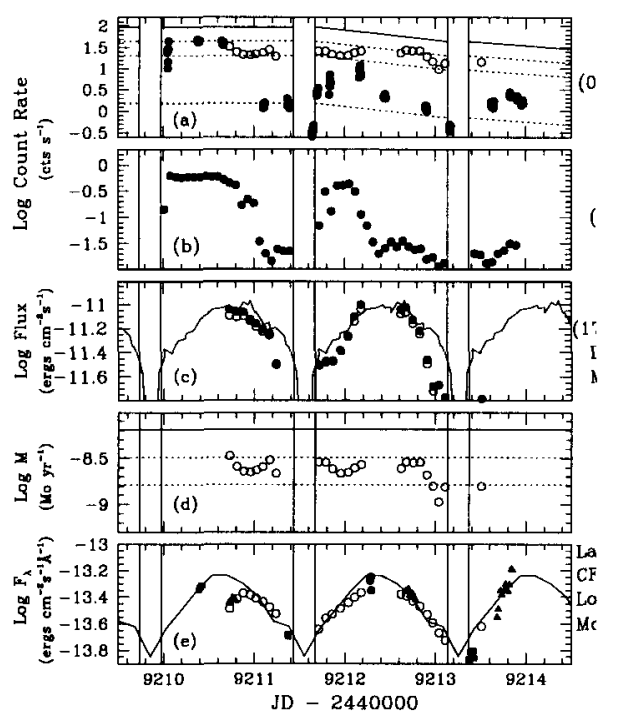

Figure 1. Model with changes in mass accretion rate alone

contribution from the star is negligible in LMXBs, it constitutes $70 \%$ of the total flux in UV and optical bands for Her $\mathrm{X}-1$.

We first fit the UV continuum while changing only $\dot{M}$. In Fig. 1 we have plotted the observed (filled circles) and model (open circles) light curves and the $\dot{M}$ values obtained by fits to the IUE data. The solid lines in Figs. 1a,d represent the highest observed X-ray fluxes and mass transfer rates, and in Figs. 1c,e the average of previous observations. The dashed lines in Fig. 1a depict X-ray fluxes at $1 / 2,1 / 4$, and $1 / 40$ th of the expected values and in Fig. 1d mass transfer rates at $1 / 2$ and $1 / 4$ of the highest observed value. Overall while we get rather good fits to the IUE continuum with changes in $\dot{M}$ only, the predicted reduction in X-ray flux is a fraction of that observed.

To further reduce the X-ray flux we can change the inclination angle of the disk with respect to our line-of-sight as is done to explain the 35-day effect. Going to a point in the 35-day cycle that totally obscures the $\mathrm{X}$-ray flux we find that disk inclination angles above $81^{\circ}$ can achieve this. We assume that the X-ray flux when unobscured is proportional to $\dot{M}$ and determine the disk inclination angle required to reduce the X-ray flux to the level observed. We then fit the IUE data for that inclination angle. We find that reductions in $\dot{M}$ by about a factor of 2 and changes in disk inclination angle of up to $20^{\circ}$ give good fits to the IUE continuum and reduces the X-ray flux appropriately (Fig. 2). 


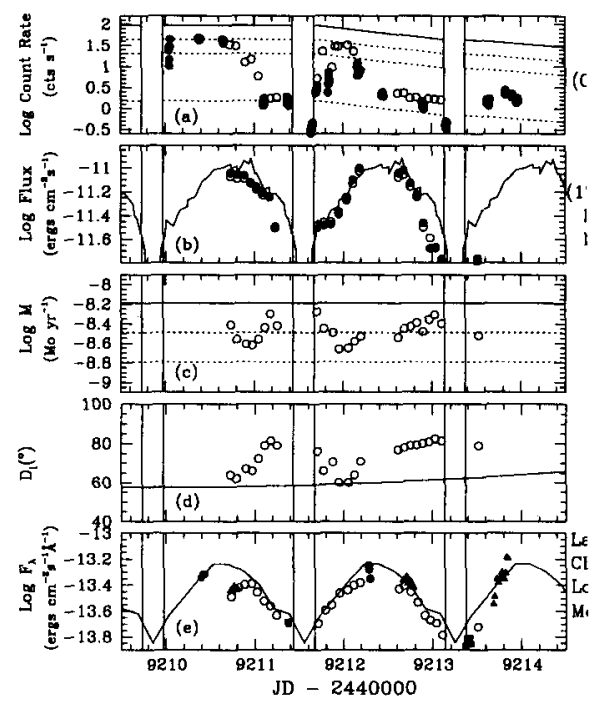

Figure 2. Model with changes in mass accretion rate and disk inclination angle

\section{Summary}

We interpret the initial drop in count rate accompanied by a change from spin-up to spin-down during our campaign in terms of a reduction in $\dot{M}$. Fits to UV/optical data show that the light curves can be reproduced by assuming a reduction in $\dot{M}$. The decrease in $\dot{M}$ is insufficient to account for the drop in X-ray flux, so disk obscuration must be taking place. The UV/optical/X-ray light curves can be reproduced by a reduction in $\dot{M}$ and accompanied changes in disk inclination angle. The change in torque on the neutron star implied by the switch from spin-up to spin-down can act to shift the disk. The lack of pulsations at energies greater than $1 \mathrm{keV}$ is consistent with the interpretation that the hard X-rays are largely occulted. The changes in disk inclination angle may be related to shifts in 35-day period. Simultaneous ultraviolet, optical, and X-ray observations over several 35-day cycles should confirm this effect.

\section{References}

Cheng, F.H., Vrtilek, S.D., Raymond, J.C., 1995, Ap. J., 452, 825

Cheng, F.H., Vrtilek, S.D., Raymond, J.C., 1996, these proceedings, p381

Ghosh, P., Lamb, F.K., 1979, Ap. J., 234, 296

Howarth, I.D., Wilson, B., 1983, MNRAS, 202, 347

Vrtilek, S.D., Mihara, T., Primini, F.A., et al., 1994, Ap. J., 436, L9

Vrtilek, S.D., Raymond, J.C., Garcia, M.R., et al., 1990, A\&A, 235, 162 